\title{
Risk Factors and Treatment Outcomes of 1,375 Patients with Testicular Leydig Cell Tumors: Analysis of Published Case Series Data
}

Fankhauser, Christian D ; Grogg, Josias Bastian ; Hayoz, Stefanie ; Wettstein, Marian S ; Dieckmann, Klaus-Peter ; Sulser, Tullio ; Bode, Peter-Karl ; Clarke, Noel W ; Beyer, Joerg ; Hermanns, Thomas

\begin{abstract}
AIMS Leydig cell tumors (LCTs) are rare but the most common non-germ cell testicular tumors. Only limited evidence exists for reliably differentiating between benign and malignant LCTs and for optimally managing the different types and stages of this rare disease. This review aims to synthesize the available evidence regarding the clinical presentation and clinicopathological characteristics associated with LCT malignancy and management. METHODS We analyzed published case series with LCTs patients. The association between clinicopathological variables and the presence of metastatic disease was assessed using regression analyses. RESULTS We included 357 reports, reviewing available data from 1,375 patients (median age: 34y). Testis-sparing surgery (TSS) was performed in 463 patients. Local recurrence after TSS occurred in 8 of 121 (7\%) patients with available follow-up information. Metastases were found in 101 patients and were most often located in the retroperitoneal lymph nodes (60\%), lungs $(38 \%)$, and/or liver $(29 \%)$. The multivariable models with or without multiple imputation predicting metastatic disease included older age, larger tumor size, the presence of any adverse factor (larger tumor diameter, necrosis, angiolymphatic invasion, pleomorphism, high mitotic index, atypia), and any protective factor (Reinke crystals, lipofuscin pigments, gynecomastia) with model AUCs of 0.93. Durable remission after resection of metastases or platinum-based chemotherapy was rarely seen. CONCLUSION Our risk tables using clinicopathological parameters can help identify patients harboring malignant tumors. These patients should undergo staging and either be followed or receive further treatment. In metastatic disease surgical and systemic treatment might result in disease control in in some patients.
\end{abstract}

DOI: https://doi.org/10.1097/JU.0000000000000705

Posted at the Zurich Open Repository and Archive, University of Zurich

ZORA URL: https://doi.org/10.5167/uzh-181720

Journal Article

Accepted Version

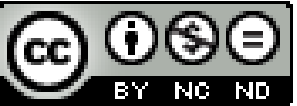

The following work is licensed under a Creative Commons: Attribution-NonCommercial-NoDerivatives 4.0 International (CC BY-NC-ND 4.0) License.

Originally published at:

Fankhauser, Christian D; Grogg, Josias Bastian; Hayoz, Stefanie; Wettstein, Marian S; Dieckmann, Klaus-Peter; Sulser, Tullio; Bode, Peter-Karl; Clarke, Noel W; Beyer, Joerg; Hermanns, Thomas (2020). 
Risk Factors and Treatment Outcomes of 1,375 Patients with Testicular Leydig Cell Tumors: Analysis of Published Case Series Data. Journal of Urology, 203(5):949-956.

DOI: https://doi.org/10.1097/JU.0000000000000705 
1 Risk factors and Treatment Outcomes of 1,375 Patients with Testicular Leydig

2 Cell Tumors: Analysis of Published Case Series Data

3 Running head: Analysis of published case series data of patients with testicular Leydig cell 4 tumors

5 Dr. Christian D. Fankhauser ${ }^{1 *} \dagger$, Josias B. Grogg ${ }^{1 *}$, Dr. Stefanie Hayoz ${ }^{2}$, Dr. Marian S. 6 Wettstein $^{1}$, Prof. Klaus-Peter Dieckmann ${ }^{3}$, Prof. Tullio Sulserr ${ }^{1}$, PD Dr. Peter-Karl Bode ${ }^{4}$, Prof. 7 Noel W. Clarke ${ }^{5}$, Prof. Joerg Beyer ${ }^{6}$, Dr. Thomas Hermanns ${ }^{1}$

$8{ }^{1}$ Department of Urology, University Hospital Zurich, University of Zurich, Zurich, Switzerland

$9 \quad 2$ SAKK Coordinating Center, Bern, Switzerland

$10 \quad{ }^{3}$ Asklepios Klinik Altona, Hamburg, Germany

$1{ }^{4}$ Institute of Pathology of Molecular Pathology, University Hospital Zurich, University of Zurich, 12 Zurich, Switzerland

$13{ }^{5}$ The Christie and Salford Royal NHS Foundation Trusts, Manchester, United Kingdom

$14{ }^{6}$ Department of Oncology, Inselspital Bern, University of Bern, Bern, Switzerland 15

$16 *$ contributed equally

$17 \dagger$ corresponding author:

18 Dr. med. Christian D. Fankhauser, Department of Urology, University Hospital Zurich, Frauenklin19 ikstrasse 10, 8091 Zurich, Switzerland. Phone: +41 4425511 11; Fax: +41 2554555

20 Email: christian.fankhauser@usz.ch

22 Keywords

23 Leydig cell cancer, Testis cancer, chemotherapy, radiotherapy, surgery, RPLND

24 Manuscript word count: 2413 (Abstract and Manuscript without declarations) 
27 Aims: Leydig cell tumors (LCTs) are rare but the most common non-germ cell testicular tumors. Only 28 limited evidence exists for reliably differentiating between benign and malignant LCTs and for opti29 mally managing the different types and stages of this rare disease. This review aims to synthesize the 30 available evidence regarding the clinical presentation and clinicopathological characteristics associated 31 with LCT malignancy and management.

32 Methods: We analyzed published case series with LCTs patients. The association between clinicopathological variables and the presence of metastatic disease was assessed using regression analyses.

34 Results: We included 357 reports, reviewing available data from 1,375 patients (median age: $34 \mathrm{y}$ ). 35 Testis-sparing surgery (TSS) was performed in 463 patients. Local recurrence after TSS occurred in 8 36 of $121(7 \%)$ patients with available follow-up information. Metastases were found in 101 patients and 37 were most often located in the retroperitoneal lymph nodes (60\%), lungs (38\%), and/or liver (29\%). 38 The multivariable models with or without multiple imputation predicting metastatic disease included 39 older age, larger tumor size, the presence of any adverse factor (larger tumor diameter, necrosis, angi40 olymphatic invasion, pleomorphism, high mitotic index, atypia), and any protective factor (Reinke crys41 tals, lipofuscin pigments, gynecomastia) with model AUCs of 0.93. Durable remission after resection 42 of metastases or platinum-based chemotherapy was rarely seen.

43 Conclusion: Our risk tables using clinicopathological parameters can help identify patients harboring 44 malignant tumors. These patients should undergo staging and either be followed or receive further treat45 ment. In metastatic disease surgical and systemic treatment might result in disease control in in some 46 patients. 
Leydig cell tumors (LCTs) are rare but are the most common non-germ cell testicular

51 tumors. LCTs usually present with a painless testicular mass and/or endocrine activity leading

52 to gynecomastia or infertility ${ }^{1}$. As the majority of LCTs are benign, most patients are cured by

53 local excision alone. The identification of an LCT with malignant potential is important for

54 detecting malignant disease at an early stage because metastatic disease is often rapidly pro-

55 gressive and usually associated with an unfavorable prognosis.

56 Given that LCTs are extremely rare, there are several unanswered questions regarding

57 the optimal management of patients with localized and metastatic disease. The aim of this study

58 was to synthesize the available information from LCT patients, including the clinical presenta-

59 tion, the clinicopathological factors associated with metastatic disease, the treatment options

60 for benign and malignant LCTs, and the outcomes of patients with metastatic spread.

61 
Search Methods and Study Selection

A literature search up to September 17, 2015, was conducted using the electronic databases MEDLINE, EMBASE, Scopus, the Cochrane Database of Systematic Reviews, and Web of Science as described in supplementary file 1.

67

\section{Statistical analysis}

Univariable logistic regression analyses were performed to identify variables potentially associated with metastatic disease. For continuous variables, receiver operating curve (ROC) analyses were performed to identify the optimal cut-off points of metastatic disease risk factors (Supplementary file 1). Due to a significant shortfall in recording of pathological variables (Supplementary Figure 1), we created two consolidated variables. One consolidated variable was defined by the presence of any of the identified adverse pathological risk factors. A second variable was defined by the presence of any protective pathological factor. We performed a first multivariable regression analysis with the consolidated variables for pathological risk factors using the original dataset without multiple imputations; the second analysis was performed after multiple imputations using chained random forests (Supplementary file 1). The multivariable models were used to calculate prediction intervals for the presence or absence of metastatic disease at initial diagnosis or during follow-up. All statistical tests were two-sided. All p-values $<0.05$ were considered statistically significant and all analyses are considered exploratory and hypothesis generating.

\section{Results}

\section{Studies}

After deduplication, 3,274 publications met the initial search criteria, of which 796 publications were eligible for full-text review after the title and abstract had been screened. After full-text screening of 534 manuscripts, 357 studies remained, with available data from 1,355 
patients (Figure 1). We also included 20 patients from our own institution, giving a total cohort of 1,375 patients of which individual patient data was available for 488 patients (Supplementary Table 1) with a median age 34 years (IQR 26-42) (Table 1).

\section{Clinical presentation}

Clinical signs and symptoms were reported in 1,056 patients: these included testicular enlargement (22\%), gynecomastia (23\%), infertility (17\%), scrotal pain (7\%), and precocious puberty (7\%). Palpable tumors were reported in 298 patients (26\%). Twenty-three percent were diagnosed incidentally during testicular ultrasonography performed for the evaluation of other conditions (e.g., infertility assessment). Serum tumor markers of germ cell cancers including alpha-fetoprotein (AFP), beta-human chorionic gonadotropin (bHCG), and lactate dehydrogenase (LDH) were elevated in $1 \%, 2 \%$, and $3 \%$ patients, respectively. The included 76 children and young adults $<18$ years mostly presented with precocious puberty $(62 \%)$ and/or testicular enlargement (46\%).

\section{Local treatment and recurrences}

The type of local treatment was mentioned in 1,342 of 1,375 patients (97\%). Radical orchiectomy was performed in $879(65 \%)$ patients, while $463(35 \%)$ underwent TSS. Nine of the latter $(2 \%)$ went on to have a completion orchiectomy due to positive surgical margins at the first operation. Follow-up information on local recurrences after TSS was available in $121 / 463$ patients (26\%). Eight recurrences $(7 \%)$ were observed after a median of 17.5 months (range 4-34 months). Five of these patients underwent further TSS, one patient had a radical orchiectomy and, for two patients, the exact local recurrence treatment was not described. One of those eight patients developed liver metastases after 107 months of follow-up, was treated with Mitotane, and showed stable disease during treatment ${ }^{2}$. Contralateral LCTs were diagnosed in a total of 17 patients (3\%) after a median of 27 months (range 1-168 months).

\section{Adjuvant therapies}


112 Adjuvant retroperitoneal lymphnode dissection (RPLND) was performed in 15 patients, of

113 which 12 patients with negative lymph nodes (LN) remained disease-free after a median of 95

114 months (Supplementary Table 2). In three cases, the histopathologic examination of the re-

115 moved LN revealed micro metastatic disease. All of those three patients with micro metastatic

116 disease additionally received chemotherapy: One patient remained disease-free at 164 months

117 after treatment with Vincristine, Actinomycin, Cyclophosphamide, and Adriblastin (VACAD),

118 whereas two patients showed progressive disease despite Cisplatin, Vinblastine, and Bleomy-

119 cin (PVB), and passed away after 51 and 31 months, respectively.

120

Metastatic spread and primary landing zone

121

Any metastatic disease at any time point was reported in 101/1040 (10\%) patients, of

whom 28 were described with only one metastatic disease site. In 6/101 the exact timing of metastatic disease in relation to diagnosis was not described whereas in 95/101 metastatic

124 spread at initial diagnosis or at disease recurrence during follow-up could be explored. At initial 125 staging, metastases were present in 30/95 patients, including the retroperitoneal LN ( $\mathrm{n}=19)$, 126 lung $(n=15)$, liver $(n=5)$, mediastinal LN $(n=4)$, kidney $(n=4)$, and brain $(n=3)$ (Figure 2). A 127 total of 65 patients developed metastases during follow-up (retroperitoneal $\mathrm{LN}(\mathrm{n}=35)$, liver $128(\mathrm{n}=24)$, lung $(\mathrm{n}=23)$, bones $(\mathrm{n}=10)$, and inguinal $\mathrm{LN}(\mathrm{n}=8)$. Metastases during follow-up were 129 diagnosed after a median of 24 months (range 1-204 months). Of note, $82 \%$ of all metastases 130 were diagnosed during the first 5 years. To determine the primary LCT landing zone, we ana131 lyzed the subgroup of the 28 patients with a single metastatic site. Retroperitoneal LN repre132 sented the most common primary landing zone, including 17 patients $(61 \%)$. Other locations

133 of single metastases were the visceral organs in 5 patients $(18 \%)$, lungs in 4 patients (14\%), 134 and bones and inguinal LN each in 1 patient (4\%).

\section{Risk factors for metastatic disease}


137 the following clinicopathological variables: age, tumor size, presence of necrosis, angiolym-

138 phatic invasion, pleomorphism, high mitotic index, atypias, Reinke crystals, lipofuscin pig-

139 ment, and gynecomastia (Table 2). To discriminate between patients with localized and meta-

140 static diseases, ROC analyses revealed ideal cut-off points for the age of 42 years and for a

141 tumor diameter of $30 \mathrm{~mm}$.

142 Both multivariable models with or without multiple imputation predicting metastatic

143 disease included tumor size, the presence of any adverse factor (larger tumor diameter, necro-

144 sis, angiolymphatic invasion, pleomorphism, high mitotic index, atypia), and any protective

145 factor (Reinke crystals, lipofuscin pigments, gynecomastia). This combination analysis re-

146 sulted in AUCs of 0.93 for both models. Age was an independent predictor of metastatic disease

147 only in the multivariable analysis using the imputed data set. All remaining variables were

148 independent predictors of metastatic disease in both models (Table 3). All potential combina-

149 tions of the four variables (largest tumor diameter, age, presence of any adverse factor and any

150 protective factor) and the associated risk for metastatic disease are shown in Figure 3 for the

151 imputed and in Supplementary Figure 2 for the original data set.

\section{Treatment of metastatic disease and outcome}

Due to the lack of therapy guidelines, many different treatment modalities have been described in the literature. Data regarding chemotherapy was available for 48 patients. The response to chemotherapy was usually poor (Supplementary Table 3). Only 3/48 (6\%) patients experienced a complete response after either Bleomycin, Etoposide, Cisplatin (BEP) or PVB,

157 but follow-up data was not provided. A partial response was described in 15/48 (31\%) patients, 158 including 5 who received Mitotane and 10 who received platinum-based chemotherapy. 
160 tunately, follow-up data was not provided (Supplementary Table 4). The most commonly per-

161 formed procedure was RPLND $(n=28)$. Information regarding clear template boundaries or

162 completeness of resection of those 28 patients was not described in most reports.

164 The effect of radiotherapy on Leydig cell tumor metastases was reported as limited, with only one published case report describing a partial response when used to treat supraclavicular me$\operatorname{tastases}^{2}$ and only two reports documented pain relief after palliative radiotherapy ${ }^{2,3}$. One

167 other case report described a favorable outcome after spinal decompression surgery and adju168 vant radiotherapy ${ }^{4}$.

\section{Discussion}

Our analysis of published case series represents the most comprehensive summary of the available literature regarding testicular LCTs. The local recurrence rate of 7\% supports the use of TSS in men with LCTs but only after appropriate counselling of the patients about the risk of recurrence and the requirement for a subsequent completion orchiectomy in case of histological risk factors. If TSS is to be used, regular testicular ultrasound should be considered for at least 3 years after surgery. To differentiate between benign and malignant LCTs, pathological workup should provide information about the presence of risk factors or protective fac-

178 tors for metastatic disease. We identified a range of these and have created a risk table based

179 on our multivariable models to predict the presence of metastasis. Due to significant missingness of individual prognostic/pathological factors in our dataset, we refrained from applying multiple imputations for each risk factor but decided, in the univariable analyses, to create

182 consolidated variables of variables that were significantly associated with metastatic disease.

183 Given this limitation, our model should be used with caution. However, in the absence of a 
clear definition of LCT malignancy, we hope that our risk table provides a clinically useful tool

185 based on the best currently available evidence and we believe that this should replace the pre-

186 viously used definition from 1985 , which was based on only 14 benign and 5 malignant cases $187 \quad 5$.

Figure 3 supports clinicians in decision-making e.g., whether further imaging or adjuvant therapy should be considered. Risk assessment can be helpful in several clinical scenarios:

190 (1) In patients with a low risk for metastatic disease (lower age, smaller tumors, absence of pathological risk factors), staging and follow-up with imaging could be omitted, and patients after TSS may only undergo ultrasound surveillance of the testis. (2) In patients with high risk

193 for metastatic disease (older age, larger tumors, presence of pathological risk factors), one

194 should consider staging and regular follow-up for at least 5 years with cross-sectional imaging of the chest, abdomen, and pelvis.

The available data does not support the regular use of adjuvant therapy for localized disease even with a high risk for metastatic disease. The fact that in two-thirds of the cases, the primary metastatic LCT landing site was the retroperitoneum adjuvant RPLND might have the potential to cure some patients with micro-metastatic disease as recently suggested ${ }^{6}$. However, as our data does not provide evidence that LCTs have a discreet step wise progression involving a specific primary landing site not clear recommendation regarding adjuvant RPLND can be given. Furthermore, chemotherapy and radiotherapy has not been shown to have curative potential in patients with metastatic LCTs. Thus, we believe that neither adjuvant chemotherapy nor radiotherapy can be recommended in patients with localized LCTs.

Patients with metastatic disease have poor prognoses, and standard treatment recommendations do not exist. Surgical resection of metastases and/or chemotherapy with BEP or

207 PVB should be discussed with the patient, as some patients were reported to have complete remission after RPLND and/or chemotherapy. Mitotane and radiotherapy represent palliative treatment options only. 
212 meta-analysis is prone to bias. Moreover, clinicopathological risk factors were often not com-

213 pletely reported. Thus, we had to use consolidated variables and/or imputations for multivari-

214 able analyses with obvious limitations. Our search criteria were designed and reviewed both

215 by clinicians and librarians and were predefined in a peer-reviewed protocol. However, it is

216 possible that not all potentially relevant studies were identified due to undetected imprecision

217 of our search strategy, which would have to be classified as another potential source of bias.

218 Give the rarity of LCT and lack of coded individual risk factors in large cancer registries more

219 conclusive answers can't be expected as shown in a recent analyses of the SEER database

220 producing only 76 LCTs $^{7}$. Moreover, the chosen cut-offs represent statistically derived cut-

221 offs, which may not represent the clinically most useful cut-offs. However, in absence of effi-

222 cacy data and decision analyses comparing surveillance versus adjuvant therapies we had to

223 apply the used methodology. Due to the absence of available retrospective or ongoing prospec-

224 tive trials and to evaluate our model, we recently opened the OrphAn Testis Histologies

225 (OATH) Registry and encourage collaborators to contribute data of patients with rare testis

226 cancer histologies (http://bit.ly/OATH-registry). Although we agree that our current recom-

227 mendations are based on a very limited level of evidence the current comprehensive analysis

228 of the published LCTs is unique and might support clinicians and patients in clinical decision

229 making.

\section{Conclusion}

231 This analysis defines clinicopathological variables associated with malignancy and suggests

232 management options in patients with testicular LCT. The presented methodology is applicable

233 in any rare cancer and may increase the knowledge in rare diseases. 
237 This analysis of published case series does not require a formal ethics approval.

\section{Consent for publication}

239 All authors consented to this publication.

240 Availability of data and material

241 The datasets used and/or analyzed during the current study are available from the correspond-

242 ing author on reasonable request.

243 Competing interests

244 The authors have nothing to disclose.

$245 \quad$ Funding

246 None

247 Authors' contributions

248 Conception and design: $\mathrm{CDF}, \mathrm{NWC}, \mathrm{JB}, \mathrm{TH}$

249 Data acquisition: JBG, CDF, PKB

250 Data analysis and interpretation: SH, MSW, KPD, TS

252 Drafting the manuscript: CDF, JBG, SH, JB, TH, PKB

253 Critical revision of the manuscript for scientific and factual content: MSW, KPD, TS, NWC

255 Statistical analysis CDF, JBG, SH, MSW

256 Supervision NWC, PKB, JB, KPD, TS, TH 
257 CDF and JG selected abstracts, extracted data and created figures. CDF and JG drafted the

258 search strategy. CDF, SH, MSW performed the statistical analysis and CDF, JB and TH drafted

259 the first manuscript draft. PKB provided histopathology support. NWC, JB, KPD, TS reviewed

260 the manuscript. All authors read and approved the final manuscript.

261 Acknowledgements

262 none

263 Figure legends

264 Figure 1 Flow chart of the study selection process

265 Figure 2 Anatomical locations of metastases in 101 patients of which the exact timing of met-

266 astatic disease in relation to diagnosis was described in $95 / 101$ patients. Whereas 30/95 patients

267 had metastatic disease at initial staging, 65/95 patients developed metastatic disease during

268 follow-up.

269 Figure 3 Point estimates and 95\% Confidence interval probabilities for metastatic disease ac-

270 cording to different clinical scenarios within the imputed data set. Adverse factor is defined by

271 the presence of any of the following variables: larger tumor diameter, necrosis, angiolymphatic

272 invasion, pleomorphism, high mitotic index, atypia). Protective factors is defined by presence

273 of any of the following variables: Reinke crystals, lipofuscin pigments, gynecomastia.

274 Table legends

275 Table 1 Patient characteristics of cases with individual patient data

276 Table 2 Univariable logistic regression analysis identifying clinicopathological predictors of

277 metastatic disease

278 Table 3 Multivariable regression analyses of clinicopathological variables predicting meta-

279 static disease 
281 Supplementary Table 1 Summary of included reports

282 Supplementary Table 2 Patients with adjuvant retroperitoneal lymph node dissection

283 Supplementary Table 3 Chemotherapy regimens and response in patients with metastatic

284 Leydig cell tumors

285 Supplementary Table 4 Surgical resection for metastatic Leydig cell tumors

286 Supplementary Table 5 Patients with metastatic Leydig cell tumors treated with radiotherapy

287 Supplementary Figure 1 Bar chart diagram describing the completeness of variable reporting

288 Supplementary Figure 2 Point estimates and 95\% Confidence interval probabilities for met289 astatic disease according to different clinical scenarios within the original dataset. Adverse

290 factor is defined by the presence of any of the following variables: larger tumor diameter, ne291 crosis, angiolymphatic invasion, pleomorphism, high mitotic index, atypia). Protective factors

292 is defined by presence of any of the following variables: Reinke crystals, lipofuscin pigments, 293 gynecomastia.

294 Supplementary File 1 Supplementary methods of three cases and review of the literature. Cancer, 58: 2116, 1986 
4. Samoladas, E. P., Anbar, A. S., Lucas, J. D. et al.: Spinal cord compression by a solitary metastasis from a low grade leydig cell tumour: a case report and review of the literature. World Journal of Surgical Oncology, 6: 75, 2008

5. Kim, I., Young, R. H., Scully, R. E.: Leydig cell tumors of the testis. A clinicopathological analysis of 40 cases and review of the literature. Am J Surg Pathol, 9: 177, 1985

6. Calaway, A. C., Tachibana, I., Masterson, T. A. et al.: Oncologic Outcomes Following Surgical

7. Osbun, N., Winters, B., Holt, S. K. et al.: Characteristics of Patients With Sertoli and Leydig Cell Testis Neoplasms From a National Population-Based Registry. Clin Genitourin Cancer, 15: e263, 2017 